\title{
Innovación metodológica y enfoques de aprendizaje en estudiantes universitarios: el caso de la carrera de ingeniería comercial en la Universidad Católica del Norte, Chile
}

\author{
Claudia A. Pezoa-Fuentes ${ }^{1 *}$ y Javier L. Mercado-Guerra ${ }^{2}$ \\ (1) Departamento de Administración, Facultad de Economía y Administración, Centro de Emprendimiento y de la Pyme \\ (CEMP), Universidad Católica del Norte, Av. Angamos 0610, Antofagasta, Chile (correo-e: cpezoa02@ucn.cl). \\ (2) Escuela de Educación, Observatorio de Investigación Interdisciplinaria en Educación para la Ciudadanía (OIIEC), \\ Vicerrectoría Académica, Universidad Católica del Norte, Av. Angamos 0610, Antofagasta, Chile \\ (correo-e: jmercado02@ucn.cl).
}

* Autor a quien debe ser dirigida la correspondencia

Recibido Oct. 17, 2019; Aceptado Dic. 18, 2019; Versión final Feb. 7, 2020, Publicado Jun. 2020

\begin{abstract}
Resumen
Esta investigación tiene por objetivo analizar la incidencia de la innovación metodológica en los enfoques de aprendizaje de los estudiantes de la carrera de ingeniería comercial de la Universidad Católica del Norte, Antofagasta, Chile. El estudio utiliza un diseño metodológico cuasi-experimental, sin preprueba con grupo de cuasi-control. En la primera fase se genera un diseño e implementación de las metodologías activas en la asignatura de administración en dos cohortes consecutivas. En la segunda fase se hace una evaluación utilizando una versión adaptada y validada del cuestionario R-SPQ-2 (Study Questionnaire Process). Se logra determinar un aumento significativo en la adopción de un enfoque profundo de aprendizaje entre los estudiantes de la segunda cohorte, quienes se beneficiaron de la planificación de las metodologías activas ajustadas a partir del diagnóstico de la primera experiencia. Este aumento significativo se produce, específicamente, en la sub-escala de estrategia profunda.
\end{abstract}

Palabras clave: docencia universitaria, innovación metodológica, enfoques de aprendizaje, administración

\section{Methodological innovation and learning approaches in university students: a case study of the commercial engineering degree at the Universidad Católica del Norte, Chile}

\begin{abstract}
This research aims to analyze the incidence of methodological innovation in the learning approaches of students enrolled in the commercial engineering degree at the Universidad Católica del Norte (Antofagasta, Chile). The study uses a quasi-experimental methodological design without a pre-test and with a quasi-control group. A design and implementation of the active methodologies in the administration subject is first generated in two consecutive cohorts. Then, an evaluation is performed using an adapted and validated R-SPQ-2 (Study Questionnaire Process). The results showed a significant increase in the adoption of a deep learning approach between the second cohort students. These students benefited from the planning of active methodologies that were adjusted using the diagnosis from their first experiences. It is concluded that the significant increase observed occurs in the subscale of deep strategy.
\end{abstract}

Keywords: university teaching, methodological innovation, learning approaches, administration 


\section{INTRODUCCIÓN}

La innovación cada vez está más presente en todas las áreas de nuestro desarrollo, propiciando procesos dinámicos en un contexto donde las nuevas generaciones son más rápidas y tecnológicas en su aprendizaje (Apple, 2013; Hernández et al., 2012). Este fenómeno constituye una realidad en las aulas universitarias en Chile (Araya-Pizarro et al., 2018; Piguave-Pérez, 2014; Ramírez y Fuentes, 2013; Yáñez-Galleguillos y SoriaBarreto, 2017) y en el mundo (Lobato y Madinabeitia, 2011; Fong et al., 2017; Yardimci et al., 2017; Rolleston et al., 2019), donde existe un gran desafío para los y las docentes. Las políticas y acciones destinadas a mejorar la equidad en el acceso a la educación superior, tienen como correlato la emergencia de diversos desafíos para los planteles universitarios, tales como el aumento en la matrícula, y, por ende, el aumento en la cantidad de estudiantes por sala de clases. Dicha problemática ha estado presente desde hace varias décadas en el debate internacional (Entwisle y Ramsden, 1983; Prosser y Trigwell, 1999), lo que se refleja en estudios y análisis que buscan determinar cómo la calidad de la docencia universitaria influye en la calidad de los aprendizajes en los estudiantes universitarios (Biggs, 2008).

Los y las docentes universitarias se ven enfrentadas a la necesidad de innovar en sus prácticas y metodologías de enseñanza, intencionado un cambio desde las prácticas más tradicionales en la enseñanza universitaria -como lo son las clases magistrales- hacia el desarrollo de metodologías activas que generen aprendizajes significativos y profundos en los estudiantes. Esta problemática ha sido abordada en estudios relacionados con estrategias activas para el desarrollo de diversas habilidades cognitivas en estudiantes universitarios (Morales-Maure et al., 2018; Rodríguez et al., 2017), así como también desde la reflexión y las buenas prácticas docentes (Yáñez-Galleguillos y Soria-Barreto, 2017) y la creatividad (Piguave-Pérez, 2014). Si bien existen diversas dificultades que impiden que los y las docentes implementen metodologías activas de enseñanza -tales como la propia resistencia al cambio, las condiciones de infraestructura y tecnología e incluso las características o perfiles de los estudiantes-, las evidencias demuestran que la innovación con metodologías activas es altamente necesaria para atender a los nuevos desafíos de la educación superior (Alba y Schumacher, 2008; Akkoyunlu y Soylu, 2008; Ibarra y Benítez, 2019; Pérez et al., 2010; Salas, 1998).

Desde esta perspectiva, el objetivo de esta investigación es analizar la incidencia de la innovación metodológica en los enfoques de aprendizaje de los estudiantes de la carrera de Ingeniería Comercial de la Universidad Católica del Norte, Antofagasta, Chile. Este objetivo se enmarca dentro de una línea de investigación en desarrollo, que tiene relación con destacar la importancia de la reflexión docente arraigada en datos empíricos que permitan iniciar procesos de mejora en la calidad de la docencia.

En relación al estudio de los enfoques de aprendizaje, las investigaciones pioneras en la temática los definen como la adaptación de estrategias de estudio que llevan a cabo los propios estudiantes para enfrentar distintas tareas asignadas por el docente (Marton y Säjlö, 1976). Investigaciones posteriores (Biggs, 1987; Biggs et al., 2001; Webster et al., 2009), señalan que los enfoques de aprendizaje consisten en procesos que surgen de las percepciones que los estudiantes tienen sobre las labores académicas propuestas por su profesor, influidas además por sus características de tipo personal. De esta manera, se sostiene que las formas de respuestas dadas por los estudiantes pueden ser el resultado de la utilización de estrategias de tipo superficial o profundo. Según Biggs (1987), el enfoque será superficial cuando exista de manera implícita una motivación extrínseca e instrumental, y será profundo cuando impere una motivación intrínseca y una preocupación por comprender. De tal modo que los estudiantes no son de por sí profundos o superficiales, sino que la o las estrategias que utilizan para su aprendizaje son el resultado de la percepción del proceso de enseñanzaaprendizaje. De esta forma, estrategias superficiales por lo general son utilizadas por los estudiantes cuando las tareas propuestas por los profesores insinúan que pueden ser resueltas de manera superficial y viceversa (Biggs, 1987).

Lo anterior se explicaría debido a que el enfoque superficial nace de la intención de liberarse de la tarea con un mínimo esfuerzo. Se utilizan unas actividades de bajo nivel cognitivo, cuando en realidad se necesitan actividades de nivel cognitivo superior para realizar la tarea de manera adecuada. Al utilizar el enfoque superficial, los estudiantes se centran en lo que Marton y Säjlö (1976), inicialmente, identificaron como signos de aprendizaje: palabras utilizadas, datos aislados, elementos tratados con independencia de otros; lo cual les impide generar un aprendizaje significativo. Al contrario, el enfoque profundo de aprendizaje se deriva de la necesidad de abordar la tarea de manera significativa; es decir, utilizando actividades que fomenten el pensamiento crítico y lleven a aplicar estrategias cognitivas de nivel superior. De este modo, el docente puede impulsar este tipo de aprendizajes por medio de métodos de enseñanza y de evaluación alineados y que apoyen las metas y resultados de aprendizaje de la asignatura. En cuanto a la elección del enfoque de aprendizaje y, de manera adicional, a la gestión del docente en el aula, se encuentra la distribución de carga académica del semestre. Si esta carga académica es muy alta, el estudiante puede llegar a asumir un enfoque superficial para poder aprobar en todas las asignaturas, principalmente por una falta de disponibilidad de tiempo para adoptar estrategias de aprendizaje profundo. Sin embargo, cuando la carga académica disminuye, o bien, en caso de que exista alguna motivación especial en la asignatura, el estudiante suele optar por un enfoque de aprendizaje profundo (Biggs, 2008). Adicionalmente, tanto el enfoque profundo como 
el superficial, pueden ser adoptados por un estudiante desde una perspectiva motivacional o estratégica; ambos sub-factores -según indican las investigaciones- estaría directamente relacionados con la forma de adopción de los enfoques profundos o superficiales (Biggs et al., 2001).

En vista a lo anterior, se pueden identificar dos factores principales que influyen en la adopción del enfoque de aprendizaje a utilizar por parte del estudiante: gestión del docente en el aula como facilitador del proceso y la carga académica del estudiante, de cuya distribución dependerá, en gran medida, el tiempo que se destine al estudio y a la adquisición de los aprendizajes. En este sentido, cabe destacar que la utilización de metodologías activas y la gestión del proceso de distribución de carga académica -según el Sistema de Créditos Transferibles (SCT-Chile)- implican un necesario cambio de paradigma por parte del docente, quién debe indagar en perspectivas beneficiosas para potenciar el aprendizaje profundo en los estudiantes universitarios.

Desde esta perspectiva, y en línea con otras investigaciones enfocadas a carreras de ingeniería comercial (Araya-Pizarro et al., 2018; Piguave-Pérez, 2014; Yáñez-Galleguillos y Soria-Barreto, 2017), esta investigación viene a aportar datos concretos respecto a la forma de abordar y evaluar el proceso de enseñanza y aprendizaje en el contexto universitario, utilizando para ello el cuestionario R-SPQ-2 validado para el contexto de la educación superior chilena (Marchant et al., 2016). De esta forma, la relevancia del estudio radica en la necesidad de identificar los enfoques de aprendizaje, proporcionando así herramientas para realizar una adecuada gestión de la docencia universitaria.

\section{MATERIALES Y MÉTODO}

La investigación se sustenta en un diseño metodológico cuasi-experimental, sin preprueba con grupo de cuasi-control (Montero y León, 2007; DeCharms, 1976). Se trata de un diseño cuasi-experimental sin preprueba, puesto que, para indagar en las percepciones de los estudiantes respecto a la asignatura cursada, no es posible aplicar una preprueba, sino que se debe realizar solamente una aplicación del cuestionario por cada muestra en la etapa final de la asignatura. Por su parte, se ha utilizado un grupo de cuasi-control, lo cual se ajusta para el caso de este diseño metodológico, en el cual se hace la aplicación del cuestionario en dos muestras que corresponden a dos cohortes de una misma asignatura, donde la primera cohorte del año 2015 opera como grupo de cuasi-control.

Un aspecto importante para el diseño metodológico de la investigación tiene que ver con evidenciar el grado de equivalencia de las dos muestras correspondientes a las cohortes de estudiantes de 2015 y 2016 de la carrera de Ingeniería Comercial de la Universidad Católica del Norte (tabla 1). La muestra está compuesta por un total de 75 estudiantes, de los cuales 37 corresponden a la cohorte 2015 y 38 estudiantes corresponden a la cohorte 2016.

La equivalencia de las muestras es evidenciada a partir de variables contextuales como el tipo de dependencia de los establecimientos educacionales que proceden los estudiantes, el tipo de enseñanza (predominantemente Científico-Humanista), el origen geográfico de los estudiantes, el sexo y el promedio obtenido en la Prueba de Selección Universitaria (PSU). A continuación, se presentan las principales características sociodemográficas y educacionales de las muestras de estudiantes.

Tabla 1: Caracterización de la muestra

\begin{tabular}{|l|l|l|}
\hline Característica & Cohorte 2015 & Cohorte 2016 \\
\hline Tipo de dependencia & $\begin{array}{l}\text { Colegios Municipales 33\%, Particular } \\
\text { Pagado 20\%, Particular subvencionado 45\% }\end{array}$ & $\begin{array}{l}\text { Colegios Municipales 42\%, Particular Pagado } \\
18 \% \text {, Particular subvencionado 39\% }\end{array}$ \\
\hline Tipo de enseñanza & $\begin{array}{l}\text { Científico Humanista-Diurno 75\%, Científico } \\
\text { Humanista Vespertino 2.5\%, Técnico } \\
\text { Comercial 10\%, Extranjero 2.5 y Técnico } \\
\text { Industrial 10\%. }\end{array}$ & $\begin{array}{l}\text { Científico Humanista-Diurno 76\%, Científico } \\
\text { Comanista Vespertino 3\%, Técnico }\end{array}$ \\
\hline Origen geográfico & $\begin{array}{l}\text { Antofagasta } 80 \% \text {, Calama 10\%, lquenico Industrial 5\%. } \\
\text { Pozo al Monte 2.5\% y Taltal 2.5\%. }\end{array}$ & $\begin{array}{l}\text { Antofagasta 68\%, Calama 16\%, lquique 5\%, } \\
\text { Mejillones 5\%, Ovalle 3\% y Taltal 3\%. }\end{array}$ \\
\hline Promedio PSU & 586.9 & 601.3 \\
\hline Sexo & $55 \%$ Mujer, 45\% Hombre & $50 \%$ Mujer, 50 Hombre \\
\hline
\end{tabular}

Como se ha señalado, ambas muestras de estudiantes presentan una clara equivalencia en diversas variables de carácter sociodemográficas y educacionales. Por ejemplo, en ambos casos alrededor del $90 \%$ de los estudiantes provienen de la Región de Antofagasta. Con respecto al tipo de enseñanza, alrededor del $75 \%$ proviene de establecimientos educacionales Científico Humanista-Diurno y el régimen escolar que agrupa la mayoría es de tipo particular subvencionados. Con respecto al promedio en la Prueba de Selección Universitaria en ambas cohortes, existen una diferencia de puntaje que no es estadísticamente significativa Para establecer esto, se realizó la prueba $U$ de Mann-Whitney (prueba no paramétrica para dos muestras independientes), tomando como variable de agrupación los puntajes PSU de las dos muestras $\left(U_{1}=607.500\right.$; $p=.311)$. 
Finalmente, para el desarrollo del proceso de investigación, se definen dos fases. La primera fase consiste en la planificación e implementación de las metodologías activas en la asignatura de Administración en los años 2015 y 2016; generándose un proceso de revisión y ajustes en la planificación de una cohorte a otra. La segunda fase de la investigación consiste en las aplicaciones del Study Process Questionnaire (SPQ), instrumento que permite indagar en los enfoques de aprendizaje en estudiantes universitarios. A continuación, se describen los elementos metodológicos que configuran cada una de estas fases del proceso de investigación.

\section{Fase de diseño e implementación}

La fase de diseño de las metodologías activas consideró un primer diagnóstico de los elementos curriculares de la asignatura Administración de la carrera de Ingeniería Comercial de la Universidad Católica del Norte. Esta asignatura aporta al Dominio I del Perfil de Egreso de la mencionada carrera, titulado Análisis del Entorno y la Organización. En ella se aborda la distinción entre los diferentes conceptos y variables para administrar y dirigir una empresa de diferentes tamaños y tipologías. Tributa a dos competencias declaradas en el perfil de egreso: 1. Analizar el entorno con una visión prospectiva que favorezca la toma de decisiones, y C2. Analizar el ámbito interno de la organización para favorecer la toma de decisiones. Busca el logro de cinco resultados de aprendizaje: A. Distinguir las diferencias entre administrar, emprender, liderar y dirigir una empresa considerando su tipología y tamaño; B. Diferenciar políticas de control general reactivo de las preventivas en empresas de tamaño medio y en un contexto de transparencia, C. Inferir evidencias y fuentes de información para ser utilizadas en la toma de decisiones a partir de análisis de casos, D. Diferenciar el impacto de las dimensiones del contexto y variables de resultados en la estructura de la organización empresarial, y E. Comparar críticamente los modelos contemporáneos de administración con los modelos clásicos.

La asignatura de Administración es de primer año, ubicada en el segundo semestre, y tiene una carga de 3 horas directas semanales y 3 horas indirectas de trabajo autónomo, sumando a 1,5 horas de ayudantía, lo cual constituye una carga de 5 créditos SCT. Realizado el análisis de los aspectos curriculares centrales de la asignatura de Administración, se desarrolló una planificación didáctica que incorporara a lo largo del semestre metodologías activas con foco en el trabajo colaborativo, considerando que se trata de grupos de tamaño medio. Para la cohorte 2015, se planificaron metodologías activas como clickers, retroalimentación inmediata (IF-AT) y estudio de casos (tabla 2). Por su parte -y a la luz de las evidencias recogidas a partir de la primera implementación-, para la cohorte 2016 se planificaron nuevamente metodologías activas, pero más focalizadas a aspectos motivacionales, las TIC y resguardando la ejecutividad de los grupos colaborativos (tabla 3).

Tabla 2: Metodologías activas cohorte 2015

\begin{tabular}{|l|l|l|l|}
\hline $\begin{array}{l}\text { Metodología o técnica } \\
\text { de enseñanza }\end{array}$ & Descripción & Unidad temática & Resultado de aprendizaje \\
\hline Clickers & $\begin{array}{l}\text { Sistema de respuesta a } \\
\text { distancia en tiempo real (Wan et } \\
\text { al., 2017) }\end{array}$ & $\begin{array}{l}\text { Dirección y } \\
\text { Liderazgo }\end{array}$ & $\begin{array}{l}\text { Distinguir las diferencias } \\
\text { entre administrar, } \\
\text { emprender, liderar y } \\
\text { distinguir una empresa } \\
\text { considerando su tipología y } \\
\text { tamaño. }\end{array}$ \\
\hline $\begin{array}{l}\text { Feedback Assessment } \\
\text { Technique) }\end{array}$ & $\begin{array}{l}\text { Técnica de retroalimentación } \\
\text { inmediata por medio de una } \\
\text { tarjeta con formato de respuesta } \\
\text { de selección múltiple (Ruiz- } \\
\text { Campo et al., 2018). }\end{array}$ & $\begin{array}{l}\text { Gestión de } \\
\text { Control }\end{array}$ & $\begin{array}{l}\text { Diferenciar políticas de } \\
\text { control general reactivo de } \\
\text { las preventivas en empresas } \\
\text { de medio y en un contexto } \\
\text { de transparencia. }\end{array}$ \\
\hline $\begin{array}{l}\text { Estudio de casos con } \\
\text { lecturas colaborativa (o } \\
\text { puzzle de Aronson) }\end{array}$ & $\begin{array}{l}\text { Técnica que estimula la } \\
\text { aplicación de conocimientos al } \\
\text { análisis o resolución de casos } \\
\text { reales del área de formación } \\
\text { (Wynn-Williams et al., 2016); se } \\
\text { trabaja con el análisis } \\
\text { colaborativo de lectura, por } \\
\text { medio de grupos iniciales que } \\
\text { generan interdependencia a } \\
\text { través de la conformación de } \\
\text { segundos grupos expertos que } \\
\text { deben comunicar su análisis al } \\
\text { grupo inicial (Aronson y Patnoe, } \\
\text { 1997). }\end{array}$ & $\begin{array}{l}\text { Toma de } \\
\text { Decisiones }\end{array}$ & $\begin{array}{l}\text { Inferir evidencia y fuentes de } \\
\text { información para ser } \\
\text { utilizadas en la toma de } \\
\text { decisiones a partir de } \\
\text { análisis de casos. }\end{array}$ \\
\hline
\end{tabular}


Las investigaciones sobre el impacto de las técnicas de enseñanza implementadas han demostrado una relación directa con el incremento en la motivación de los estudiantes. Esto fue posible evidenciar con mayor énfasis en el uso de clickers a partir del 2015. Esta técnica su utilizó en la fase de inicio de la clase con la finalidad de realizar un diagnóstico por medio de preguntas relacionadas con la unidad temática, por ejemplo, sobre el rol del administrador, los roles de liderazgo, entre otra. Se logró evidenciar un mayor interés de parte de los estudiantes por comprender el contenido expuesto, lo cual quedó evidenciado al finalizar la clase, cuando se realiza nuevamente una evaluación en tiempo real sobre el contenido desarrollado.

Tabla 3: Metodologías activas cohorte 2016

\begin{tabular}{|l|l|l|l|}
\hline $\begin{array}{l}\text { Metodología o técnica } \\
\text { de enseñanza }\end{array}$ & Descripción & Unidad temática & Resultado de aprendizaje \\
\hline Clickers & $\begin{array}{l}\text { Sistema de respuesta a } \\
\text { distancia en tiempo real (Wan et } \\
\text { al., 2017) }\end{array}$ & $\begin{array}{l}\text { Dirección y } \\
\text { Liderazgo }\end{array}$ & $\begin{array}{l}\text { Distinguir las diferencias } \\
\text { entre administrar, } \\
\text { emprender, liderar y } \\
\text { distinguir una empresa } \\
\text { considerando su tipología y } \\
\text { tamaño. }\end{array}$ \\
\hline Powtoon & $\begin{array}{l}\text { Herramienta que permite crear } \\
\text { presentaciones incorporando } \\
\text { animaciones con videos y audio } \\
\text { (Pais et al., 2017). }\end{array}$ & $\begin{array}{l}\text { Dirección y } \\
\text { Liderazgo }\end{array}$ & $\begin{array}{l}\text { Distinguir las diferencias } \\
\text { entre administrar, } \\
\text { emprender, liderar y } \\
\text { distinguir una empresa } \\
\text { considerando su tipología y } \\
\text { tamaño. }\end{array}$ \\
& $\begin{array}{l}\text { tamorar críticamente los } \\
\text { Comparar } \\
\text { modelos contemporáneos de } \\
\text { administración con los } \\
\text { modelos clásicos }\end{array}$ \\
\hline Foros de discusión & $\begin{array}{l}\text { Herramienta no presencial que } \\
\text { permite desarrolla una discusión } \\
\text { asincrónica entre el docente y el } \\
\text { grupo curso sobre un tema en } \\
\text { particular (Galikyan y Admiraal, } \\
\text { 2019). }\end{array}$ & $\begin{array}{l}\text { Tipologías de } \\
\text { Empresas }\end{array}$ & $\begin{array}{l}\text { control general reactivo de } \\
\text { las preventivas en empresas } \\
\text { de medio y en un contexto } \\
\text { de transparencia. }\end{array}$ \\
\hline
\end{tabular}

Para la implementación del año 2016, se realizaron ajustes a la planificación didáctica de la asignatura, incorporando con mayor énfasis aspectos relacionados con herramientas tecnológicas, además de mantener el uso regular de clickers en la sala de clases. En esta segunda implementación, se evidenció la potencialidad motivadora de la herramienta informática Powtoon, la cual se utilizó a partir de la realización de un trabajo en grupo de 6 estudiantes, quienes debían crear una propuesta animada sobre los estilos de dirección y liderazgo, enfocándose en la descripción de un líder y sus tareas. Las animaciones eran compartidas por la plataforma institucional para recibir la retroalimentación de sus pares de curso. En esta experiencia se evidencia un incremento de la motivación y compromiso de los estudiantes, evidenciado en el estricto cumplimiento de los plazos establecidos, además de un incremento en las interacciones en los foros de discusión.

A nivel comparativo entre las experiencias de implementación con las cohortes 2015 y 2016, resulta necesario destacar que en la primera implementación no se realizó una planificación didáctica sustentada en los resultados de aprendizaje, sino que fue a través del análisis de las unidades temáticas de la asignatura. En cambio, para segunda implementación se realizaron ajustes de acuerdo al diagnóstico que se levantó el 2015 y se trabajó en una planificación orientada por los resultados de aprendizaje de la asignatura.

Un aspecto común en ambas implementaciones es la planificación de una enseñanza centrada en el trabajo colaborativo, el cual tiene como fundamento pedagógico el que los y las estudiantes puedan crear significados y desarrollar aprendizajes por medio del consenso entre compañeros (Barkley et al., 2007); a diferencia del aprendizaje cooperativo que apunta a que el docente conserve el doble papel de facilitador y figura de autoridad como experto en el aula. Según las investigaciones en el ámbito de la educación superior, el desarrollo de un aprendizaje centrado la colaboración aporta a elevar el rendimiento académico de los y las estudiantes, ayuda a generar relaciones que permitan establecer una comunidad de aprendizaje y permite generar experiencias saludables para el desarrollo social, psicológico y cognitivo (Barkley et al., 2007; Johnson et al., 1999; Roselli, 2016); todo aquello enmarcado en el enfoque que concibe al fenómeno del aprendizaje como un fenómeno eminentemente social. En este sentido, las metodologías activas implementadas consideraron los siguientes elementos centrales dentro del aprendizaje colaborativo: la interdependencia positiva entre los estudiantes, la interacción grupal, la integración social, la evaluación grupal y la responsabilidad individual. 


\section{Fase de evaluación}

La segunda fase de la investigación consistió en realizar una indagación en los enfoques de aprendizaje adoptados por los estudiantes que cursaban la asignatura de Administración en la carrera de Ingeniería Comercial de la Universidad Católica del Norte. Para ello, se aplicó el cuestionario Study Process Questionnaire (SPQ), el cual permite recoger evidencias respecto al enfoque de aprendizaje que los estudiantes despliegan al momento de afrontar su aprendizaje en la asignatura. En este sentido, y tal como ya se ha señalado, el enfoque de aprendizaje no debe concebirse de manera alguna como una categoría fija ni estable en el grupo de estudiantes, sino que hace referencia más bien a las estrategias asumidas por el grupo de estudiantes en el contexto de aprendizaje presentado y propiciado por el o la docente de cada asignatura (Biggs, 1999; Ramsden, 2003). El análisis de los enfoques de aprendizaje desarrollado por estudiantes universitario, forma parte de un modelo evaluativo más amplio que también se relaciona con las experiencias de aprendizaje. Dicho modelo ha sido denominado como el Modelo de las 3P: Presagio, Proceso y Producto (Bigss, 2008; Marchant et al., 2016), y establece como explicación que "la percepción de buena docencia y metas y objetivos claros [escalas presentes en el ámbito de la experiencia de aprendizaje] presentan correlaciones directas con el uso de un enfoque profundo de aprendizaje, a diferencia de la percepción sobre la carga académica de trabajo y la evaluación, que presentan correlaciones con el aprendizaje superficial" (Marchant et al., 2016).

Si bien las variables implicadas y sus correlaciones permiten obtener una explicación más completa sobre las formas en que se producen los aprendizajes de calidad en la educación superior, en este trabajo se aplicó únicamente el segmento relacionado con el R-SPQ-2 (SPQ versión revisada de dos factores) validado para el contexto universitario chileno (Marchant et al., 2016), con el objetivo de evidenciar cómo pueden incidir las metodologías activas en el desarrollo de un enfoque profundo. Cabe señalar que el R-SPQ-2 es un instrumento que cuenta con muchas evidencias recientes de confiabilidad y validez, entregadas a partir de las diversas adaptaciones realizadas en diferentes contextos culturales, tales como Asia (Gan et al, 2019; Malik et al., 2019), África (Rolleston et al, 2019), Oceanía (Vaughan, 2016, 2018) Europa (Zakariya, 2019, Fernández-Polvillo y Arquero, 2015), Medio Oriente (McLaughlin y Durrant, 2017; Shaik et al., 2017) y Latinoamérica (Marchant et al., 2016, 2017; Vergara-Hernández, et al., 2019).

EI R-SPQ-2 cuenta con dos dimensiones (enfoque profundo y enfoque superficial) y cuatro sub-escalas (motivación profunda, estrategia profunda, motivación superficial y estrategia superficial). El cuestionario cuenta con 20 ítems (10 para cada dimensión y, dentro de ellas, cinco para cada sub-escala) ante los cuales cada participante debe responder a una escala tipo Likert cuyo rango va desde 1 (nunca o casi nunca) a 5 (siempre o casi siempre). Algunos ejemplos de reactivos de la escala enfoque profundo son: "cuando estudio un tema de este curso, no estoy conforme hasta que le dedico tiempo suficiente para formar mis propias conclusiones", "autoevalúo mis conocimientos sobre temas relevantes de este curso, hasta que los entiendo por completo" y "me esfuerzo por revisar la mayor parte del material de estudio recomendado para las clases de este curso". Por su parte, algunos ejemplos de reactivos de la escala enfoque superficial son: "puedo aprobar la mayoría de las evaluaciones de este curso memorizando los temas más relevantes, más que tratando de comprender", "en este curso generalmente me limito a estudiar sólo lo que establece el profesor, porque creo que es innecesario hacer el trabajo extra y "el(la) profesor(a) de este curso no debería esperar que los estudiantes dediquen mucho tiempo a estudiar contenidos que se sabe que no serán considerados en las evaluaciones".

Los resultados obtenidos a través de la aplicación del SPQ deben ser interpretados por medio de un método de comparación de los puntajes obtenidos en ambas dimensiones del cuestionario por cada persona o grupo (Prosser y Trigwell, 2006). Junto con ello, en este caso se realizó un análisis de algunos estadísticos descriptivos por cada cohorte, para establecer luego comparaciones de resultados y evidenciar la posible existencia de diferencias significativas los grupos. Todas la mediciones y test estadísticos se realizaron con el software IBM SPSS v.21.

El cuestionario SPQ fue aplicado de forma presencial durante los meses de noviembre de 2015 y 2016 , coincidiendo con la última etapa del desarrollo de la asignatura. El cuestionario fue tomado por una persona externa al proceso de investigación, y se aplicó bajo mecanismos de confidencialidad y anonimato. Para indagar en la confiabilidad del instrumento se aplicó el coeficiente Alfa de Cronbach a las dos dimensiones que componen el SPQ, y se obtuvieron resultados aceptables y muy similares a los obtenidos en la validación realizada por Marchant et al. (2016). En este caso, para la dimensión enfoque profundo, se obtuvo un índice de 0.792 y para la dimensión enfoque superficial se obtuvo un índice de 0.756 .

\section{RESULTADOS Y DISCUSIÓN}

Las evidencias comparativas entre las cohortes 2015 y 2016 nos demuestran un claro predominio del enfoque profundo en ambos grupos de estudiantes (tabla 4). Así mismo, se puede evidenciar un incremento en la media de la escala enfoque profundo en la cohorte 2016, en comparación a media de la cohorte 2015. Esta 
diferencia en el incremento de la media de la escala enfoque profundo resulta ser estadísticamente significativa, al aplicar una prueba T para muestras independientes $(p=.024)$. Aquello permite establecer que la cohorte 2016 adopta un enfoque de aprendizaje significativamente más alto que la cohorte 2015. Por otra parte, también se puede apreciar una disminución en la media de la escala enfoque superficial en la cohorte 2016, como consecuencia del incremento en la media de la escala de enfoque profundo. Sin embargo, en este caso, la disminución en la media no resulta ser estadísticamente significativa $(p=.171)$.

Tabla 4: Resultados escalas enfoque profundo y enfoque superficial. Nota: M=media; DT: desviación típica; $\mathrm{N}$ : tamaño muestra.

\begin{tabular}{|l|c|c|c|c|c|c|c|c|}
\hline \multirow{2}{*}{ Escala } & \multicolumn{3}{|c|}{2015} & \multicolumn{3}{|c|}{2016} & \multirow{2}{*}{$t$} & \multirow{2}{*}{$p$} \\
\cline { 2 - 9 } & $\mathrm{M}$ & $\mathrm{DT}$ & $\mathrm{N}$ & $\mathrm{M}$ & $\mathrm{DT}$ & $\mathrm{N}$ & & .024 \\
\hline Enfoque Profundo & 3.25 & .479 & 37 & 3.51 & .505 & 38 & -2.297 & .171 \\
\hline Enfoque Superficial & 2.75 & .548 & 37 & 2.56 & .599 & 38 & 1.384 & .171 \\
\hline
\end{tabular}

Ahora bien, cabe destacar que la estrategia de análisis de los enfoques de aprendizaje también considera la existencia de cuatro sub-escalas: motivación profunda, estrategia profunda, motivación superficial y estrategia superficial (Biggs et al., 2001). Desde este punto de vista, la sub-escala motivación profunda expresa un incremento en la media entre la cohorte 2015 a la 2016, que va de 3.38 a 3.59. Igual caso ocurre la escala estrategia profunda, con una media de 3.13 para la cohorte 2015 y de 3.44 para la cohorte 2016 (tabla 5). Dentro del aumento en las medias de ambas sub-escalas, solamente la correspondiente la estrategia profunda presenta una diferencia estadísticamente significativa $(p=.022)$. En este sentido, se puede establecer que el incremento en los enfoques de aprendizaje profundo en la cohorte 2016, se limitan a un tipo aprendizaje profundo de tipo estratégico.

Tabla 5: Resultados sub-escalas enfoques de aprendizaje

\begin{tabular}{|l|c|c|c|c|c|c|c|c|}
\hline \multirow{2}{*}{ Sub-escala } & \multicolumn{3}{|c|}{2015} & \multicolumn{3}{|c|}{2016} & \multirow{2}{*}{$t$} & \multirow{2}{*}{$p$} \\
\cline { 2 - 8 } & $\mathrm{M}$ & $\mathrm{DT}$ & $\mathrm{N}$ & $\mathrm{M}$ & $\mathrm{DT}$ & $\mathrm{N}$ & \\
\hline Motivación profunda & 3.38 & .509 & 37 & 3.59 & .565 & 38 & -1.741 & .086 \\
\hline Estrategia profunda & 3.13 & .560 & 37 & 3.44 & .577 & 38 & -2.338 & .022 \\
\hline Motivación superficial & 2.57 & .666 & 37 & 2.44 & .725 & 38 & .813 & .419 \\
\hline Estrategia superficial & 2.94 & .523 & 37 & 2.71 & .619 & 38 & 1.704 & .093 \\
\hline
\end{tabular}

Por su parte, en cuanto las sub-escalas motivación superficial y estrategia superficial, se puede evidenciar una disminución en las medias en la cohorte 2016. En el caso la sub-escala motivación superficial, la cohorte 2015 presenta una media de 2.57, y la cohorte 2016 presenta una media de 2.44 . Por su parte, en la subescala estrategia superficial, la cohorte 2015 presenta una media de 2.94, mientras que la cohorte 2016 presenta una media de 2.71. En este caso, las diferencias entre las medias de ambas sub-escalas no son significativas ( $p=.419$ y $p=.093$, respectivamente). Estos resultados permiten establecer que, tanto el aumento significativo en la escala enfoque profundo como también en la sub-escala estrategia profunda, guardan una estrecha relación con los ajustes a la planificación didáctica y la implementación de metodologías activas focalizadas al desarrollo del trabajo colaborativo y por medio del uso de TIC.

Se pueden apreciar varios aspectos relevantes a partir de los datos arrojados por esta muestra longitudinal de dos cohortes de estudiantes. En primer lugar, los casos de estudiantes con enfoque profundo son predominantes en ambas cohortes, sin embargo, los puntajes medios de esta escala son relativamente moderados. Estos hallazgos se condicen con lo expresado por las investigaciones internacionales (Biggs, 2008), las cuales indican que en el caso de estudiantes universitarios existe la tendencia a asumir su aprendizaje con un enfoque profundo moderado, debido, entre otras cosas, a la alta carga académica que suelen tener dentro del contexto universitario. Esta situación tiende a impulsar la adopción de estrategias de aprendizaje de carácter estratégico que permitan el logro de los resultados académicos esperados, pero que a su vez impide la adquisición de un tipo de aprendizaje profundo que logre finalmente generar un aprendizaje significativo. En el caso analizado, podemos ver que existe un aumento significativo, justamente, en la subescala estrategia profunda, y no así en la sub-escala motivación profunda.

Desde esta perspectiva, es posible establecer alguna relación en el desarrollo del enfoque profundo con el cambio en los tipos de metodologías activas y los tipos de actividades propuestas por el docente, puesto que durante la segunda experiencia del año 2016 se realizó un ajuste reflexivo de las actividades, estableciendo una prioridad en aquellas que resultaron más efectivas en relación a la experiencia anterior del año 2015. En concreto, con la cohorte del año 2016 se implementaron metodologías activas y estrategias basadas en Powtoon y foros de discusión. En comparación al año 2015, durante la segunda implementación se fortaleció el uso de TIC y se apuntó a un mayor protagonismo de los estudiantes en el desarrollo de las actividades, responsabilizándolos de su propio aprendizaje. La técnica sustentada en la metodología activa que sí se mantuvo en el trabajo con ambas cohortes, fue el desarrollo de las clickers en la sala de clase, para fortalecer la orientación de las técnicas de enseñanza hacia las TIC. 
Además de las evidencias estadísticas presentadas, otra estrategia de retroalimentación sobre la propia práctica docente es la indagación en los resultados obtenidos a partir de la encuesta de evaluación institucional que desarrolla la Universidad Católica del Norte. Esta encuesta es una evaluación de término dirigida hacia el docente y su práctica, donde se recoge información desde la perspectiva de los estudiantes, en base a dimensiones como metodología docente, pertinencia curricular, material de enseñanza, proceso de evaluación y vínculo estudiante-profesor.

Esta evaluación docente se aplica antes de finalizar cada semestre académico en la Universidad, y constituye uno de las principales herramientas institucionales para el análisis y evaluación de la calidad de la docencia. Si bien se trata de una evaluación dirigida hacia las funciones del docente, cabe destacar que su utilización responde más bien a la gestión de los procesos académicos para la toma de decisiones, a fin de establecer instancias de apoyo en aquellas asignaturas críticas. Si analizamos dicha evaluación institucional, podemos evidenciar que, si bien en el año 2015 existe una evaluación con alto porcentaje de valoraciones en todas las dimensiones, para el año 2016 las dimensiones que refieren a la metodología docente y al material de enseñanza, tiene una valoración sumativa más alta comparativamente. Esta mayor valoración de las dimensiones relacionadas con la metodología y los materiales, puede tener relación con el mayor desarrollo del enfoque profundo y especialmente con el aumento significativo en sub-escala estrategia profunda.

Adicionalmente, cabe destacar que el rendimiento académico promedio de la asignatura de la cohorte del año 2015 fue de un 4,1. En cambio, el de la cohorte del año 2016 fue de una nota 4,9, aumentando considerablemente. Si bien el rendimiento académico no es un indicador decidor en la evaluación de los enfoques de aprendizaje, sí constituyen también un elemento más a considerar dentro del análisis general sobre los enfoques de aprendizaje adoptados por los estudiantes. Sin embargo, en esta misma línea, un aspecto relevante a considerar en un futuro proceso de indagación en la práctica docente universitaria, es evaluar el grado de alineación que tienen los procesos de implementación de las metodologías activas y los tipos de evaluación que se proponen por parte del docente. Está demostrado que la alineación entre metodología de enseñanza y estrategias de evaluación, constituye un aspecto crucial en la calidad de la docencia, y, en este entendido, resultaría relevante preguntarse cómo los tipos de evaluación propuestos por el docente inciden en la adopción de enfoques de aprendizaje profundo.

En definitiva, se puede evidenciar que el ajuste de las metodologías activas desarrolladas en el año 2016 llega a incidir en una mayor adopción del enfoque profundo y especialmente tiene incidencia en el aumento significativo de los puntajes asociados a la sub-escala estrategia profunda. El desafío entonces es intentar generar procesos de planificación de la docencia que respondan al contexto y a los perfiles cada vez más diferenciados de los estudiantes, para lograr alcanzar una mayor incidencia de los enfoques de aprendizaje profundos y motivacionales entre los estudiantes. Así mismo, otro desafío importante es trasnversalizar entre la propia carrera, entre las diferentes unidades académicas y la propia institución de educación superior, la estrategia de evaluación de los enfoques de aprendizaje, especialmente para levantar información sobre la efectividad o incidencia que tiene la implementación de metodologías activ as en la calidad de los aprendizajes de los estudiantes.

\section{DISCUSION FINAL}

El desarrollo de la implementación de metodologías activas en la docencia universitaria se hace cada vez más frecuente. Sin embargo, un aspecto siempre deficitario es la indagación sobre la efectividad en los procesos de innovación en la docencia. En este entendido, la presente investigación ha buscado establecer una metodología de levantamiento de información que permita al docente tener indicios sobre cómo los estudiantes perciben su enseñanza, y especialmente, cómo las estrategias metodológicas del docente permiten el desarrollo de enfoques profundos de aprendizaje, es decir, pueden llegar propiciar el aprendizaje significativo.

En este sentido, a partir de los resultados de la investigación se logra identificar el enfoque de aprendizaje que expresan los estudiantes de la carrera de Ingeniería Comercial de la Universidad Católica del Norte, Antofagasta, evidenciado un claro incremento en la escala de enfoque profundo y también en la sub-escala estrategia profunda, a partir de la segunda experiencia de implementación de metodologías activas del año 2016; todo ello fruto de un proceso de reflexión y ajustes de la planificación inicial. Estos resultados permiten dar cumplimiento al objetivo inicial, pudiendo establecer que efectivamente la innovación metodológica tiene incidencia en los estudiantes a partir de una mayor incidencia del enfoque de aprendizaje profundo, y más específicamente, en el aprendizaje profundo estratégico. Es así como los resultados demuestran que la innovación metodológica puede incidir positivamente en un mayor desarrollo del enfoque de aprendizaje profundo.

La relevancia de este estudio radica en la importancia del análisis de los enfoques de aprendizaje como estrategia de evaluación y mejora continua en la docencia universitaria, puesto que con esta información es posible obtener mejoras significativas en los procesos de enseñanza y aprendizaje. El contar con un instrumento que permite establecer el enfoque de aprendizaje que utilizan los estudiantes frente a las 
actividades de enseñanza desarrolladas por el docente, presenta una oportunidad de evaluación de la labor docente enfocada en las actitudes y formas de afrontar el aprendizaje por parte de los propios estudiantes. Si este tipo de evaluación se triangula además con la evaluación docente institucional que se implementa en las universidades, el docente pude contar con un insumo muy relevante para su desarrollo docente, permitiendo profesionalizar su labor y propiciar procesos de mejora continua en su enseñanza.

Las metodologías activas deben ser planificadas, articuladas con objetivos de aprendizaje bien definidos, para así fomentar el desarrollo del aprendizaje profundo. Dentro de la práctica docente, es posible utilizar varias metodologías activas para obtener mejoras en los aprendizajes de los estudiantes, así como también en la comunicación y participación de los mismos. Sin embargo, y más allá de la utilización y puesta en práctica de las metodologías activas, también es necesario definir mecanismos claros que permitan al docente indagar en la incidencia o efectividad de las metodologías activas desarrolladas a partir de su práctica. Es por ello que esta investigación espera contribuir con elementos que permitan avanzar en la definición de mecanismos que permitan al docente universitario reflexionar sobre su propia práctica e iniciar procesos de indagación y mejora continuar, siempre en búsqueda de prácticas docentes que aporten a la calidad de los aprendizajes de sus estudiantes.

\section{CONCLUSIONES}

De acuerdo al trabajo presentado y a los resultados obtenidos, se pueden plantear las siguientes conclusiones principales:

1.- De acuerdo al objetivo de investigación plantado, se logra determinar que la implementación de metodologías activas planificadas y sustentadas en un diagnóstico previo, logran incidir en un aumento significativo en la adopción de un enfoque profundo de aprendizaje entre los estudiantes. Junto con ello, se logra determinar un aumento significativo en la sub-escala estrategia profunda de aprendizaje, lo cual se condice con la orientación hacia el cumplimiento de las tareas, en el marco de las exigencias de carga académica que experimentan usualmente los estudiantes universitarios.

2.- Se logra observar una disminución en la adopción de enfoques de aprendizaje superficial a partir de la implementación de metodologías activas con uso predominante de TIC. Sin embargo, en este caso las disminuciones no son estadísticamente significativas, más allá de que estos datos se explican por la mayor incidencia del enfoque profundo en la cohorte 2016.

3.- Finalmente, se deja en evidencia la utilidad y confiabilidad del cuestionario R-SPQ-2 para la evaluación y retroalimentación de la propia práctica docente, y específicamente, para indagar en la incidencia que tienen las implementaciones de metodologías docentes activas en los enfoques de aprendizaje de estudiantes universitarios en el contexto chileno.

\section{REFERENCIAS}

Akkoyunlu, B. y Soylu, M.Y., A study of student's perceptions in a blended learning environment based on different learning styles, Journal of Educational Technology \& Society, 11(1), 183-193 (2008)

Alba, F. y Schumacher, C., Evaluación del aprendizaje universitario. Educación y educadores, 11(2), 91-105 (2008)

Apple, K.J., Embedding assessment into college-level psychology courses; in Assessing teaching and learning psychologist, pp. 35-43, Waswordth, Cengage Learning, Belmont, USA (2013)

Araya-Pizarro, S.C., Varas-Madrid, C.P., y Rojas-Escobar, L.E., Atributos preferidos por los estudiantes de ingeniería comercial en la enseñanza de la asignatura Administración de Empresas, https://doi.org/10.4067/s071850062018000100077, Formación Universitaria, 11(1), 77-86 (2018)

Aronson, E. y Patnoe, S., The jigsaw classroom. Building cooperation in the classroom, Longman, USA (1997).

Barkley, E.F., Cross, K.P. y Major C.H., Técnicas de aprendizaje colaborativo. Manual para el profesorado universitario, Morata, Madrid (2007)

Biggs, J.B., Kember, D. y Leung, D.Y.P., The revised two-factor Study Process Questionnaire: R-SPQ-2F, https://doi.org/10.1348/00070990115843, British Journal of Educational Psychology, 71, 133-149 (2001)

Biggs, J.B., Calidad del aprendizaje universitario, Narcea, Madrid (2008)

Biggs, J.B., The Study Process Questionnaire (SPQ): Manual. Australian Council for Educational Research, Hawthorn (1987)

DeCharms, R., Enhancing motivation: change in the classroom, Irvington, New York, USA (1976).

Entwistle, N.J., y Ramsden, P., Understanding student learning, Croom Helm, London (1983)

Fernandez-Polvillo, C. y Arquero, J.L., Approaches to learning: an analysis of basic psychometric properties of the Spanish versions of three short questionnaires, Educade. Revista de Educación en Contabilidad Finanzas y Administración de Empresas, 6, 23-33 (2015) 
Fong, S.M., Chu, S.K., y otros tres autores, Incorporating wiki technology in a traditional biostatistics course: effects on university students' collaborative learning, approaches to learning and course performance, https://doi.org/10.28945/3794, Journal of Information Technology Education Research, 16, 285-299 (2017)

Galikyan, I., y Admiraal, W., Students' engagement in asynchronous online discussion: The relationship between cognitive presence, learner prominence, and academic performance, https://doi.org/10.1016/j.iheduc.2019.100692, Internet and Higher Education, 43, 1-9, (2019)

Gan, Z., He, J., y Mu, K., Development and validation of the Assessment for Learning Experience Inventory (AFLEI) in Chinese higher education, https://doi.org/10.1007/s40299-019-00435-7, The Asia-Pacific Education Researcher, 28(5), 371-385 (2019)

Goh, P.S., Wong, K.T., y Hamzah, M., Re-structuring the Revised Two-Factor Study Process Questionnaire (R-SPQ-2F) in the context of pre-service teachers in Malaysia, Pertanika Journal of Social Sciences and Humanities, 25, 805-821 (2017)

Hernández, F., Arán, A. y Salmerón, H., Enfoques de aprendizaje y metodologías de enseñanza en la universidad, Revista Iberoamericana de Educación, 60(3), 1-12 (2012)

Ibarra, S.V., y Benítez, A.A., El diálogo en el aula con estudiantes de nivel superior en clases de humanidades para ingeniería, https://doi.org/10.4067/S0718-50062019000100055, Formación Universitaria, 12(1), 55-64 (2019)

Johnson, D.W., Johnson, R.T., y Holubec, E.J., El aprendizaje cooperativo en el aula, Paidós, Buenos Aires (1999)

Lobato, C., y Madinabeitia, A., Perfiles motivacionales del profesorado ante la formación en metodologías activas en la universidad, https://doi.org/10.4067/s0718-50062011000100006, Formación Universitaria, 4(1), 37-48 (2011)

Malik, A.A., Khan, R.A., y otros cuatro autores, Assessing reliability and validity of Revised Biggs Two-Factor study process questionnaire to measure learning approaches among undergraduate medical students in Lahore, Pakistan, Journal of the Pakistan Medical Association, 69(3), 337-342 (2019)

Marchant, J., Fauré, J. y Abricot, N., Adaptación y validación del SPQ y el CEQ para el estudio de la formación en docencia universitaria en el contexto chileno, Psykhe, 25(2), 1-18 (2016)

Marchant, J., González, C., y Fauré, J., The impact of a university teaching development programme on student approaches to studying and learning experience: evidence from Chile, https://doi.org/10.1080/02602938.2017.1401041, Assessment \& Evaluation in Higher Education, 43(5), 697-709 (2017)

Marton, F., y Säjlö, R., On qualitative difference in Learning-l: outcome and process, British Journal of Education Psychology, 46, 4-11 (1976)

McLaughlin, J., y Durrant, P., Student learning approaches in the UAE: the case for the achieving domain. https://doi.org/10.1080/07294360.2016.1176998, Higher Education Research \& Development, 36(1), 158-170 (2016)

Montero, I. y León, O.G., A guide for naming research studies in Psychology, International Journal of Clinical and Health Psychology, 7(3), 847-862 (2007)

Morales-Maure, L., García-Marimón, O., y otros dos autores, Habilidades cognitivas a través de la estrategia de aprendizaje cooperativo y perfeccionamiento epistemológico en matemática de estudiantes de primer año de universidad, https://doi.org/doi:10.4067/S0718-50062018000200045, Formación Universitaria, 11(2), 45-56 (2018)

Rioseco, M.H., Nogues, F.P., y Ramírez, B., Incorporating powtoon as a learning activity into a course on technological innovations as didactic resources for pedagogy programs, https://doi.org/10.3991/ijet.v12i06.7025, International Journal of Emerging Technologies in Learning, 12(6), 120-131 (2017)

Pérez, M.V., Valenzuela, M.F., y otros tres autores, Disposición y enfoques de aprendizaje en estudiantes universitarios de primer año, https://doi.org/10.11144/Javeriana.upsy10-2.deae, Universitas Psychologica, 10(2), 441-449 (2011).

Piguave-Pérez, V.R., Importancia del desarrollo de la creatividad para los estudiantes de la carrera de Ingeniería Comercial desde el proceso de enseñanza-aprendizaje, Educación, 23(44), 29-47 (2014)

Prosser, M., y Trigwell, K. (2006). Confirmatory factor analysis of the Approaches to Teaching Inventory, https://doi.org/10.1348/000709905X43571, British Journal of Educational Psychology, 76, 405-419.

Prosser, M., y Trigwell, K., Understanding learning and teaching. The experience in higher education, SRHE, Open University Press Buckingham (1999)

Ramírez, P.E., y Fuentes, C.A., Felicidad y rendimiento académico: efecto moderador de la felicidad sobre Indicadores de selección y rendimiento académico de alumnos de ingeniería comercial, https://doi.org/10.4067/s071850062013000300004, Formación Universitaria, 6(3), 21-30 (2013)

Rodríguez, A.B., Ramírez, L.J. y Fernández, W., Metodologías activas para alcanzar el comprender, https://doi.org/10.4067/s0718-50062017000100009, Formación Universitaria, 10(1), 79-88 (2017)

Rolleston, C., Schendel, R. y Grijalva, A.M., Assessing "approaches to learning” in Botswana, Ghana and Kenya, https://doi.org/10.1177/1745499919829216, Research in Comparative and International Education, 14(1), 118-140 (2019)

Roselli, N.D., El aprendizaje colaborativo: bases teóricas y estrategias aplicables en la enseñanza universitaria, https://doi:10.20511/pyr2016.v4n1.90, Propósitos y Representaciones, 4(1), 219-280 (2016) 
Ruiz-Campo, S., Soria-Barreto, K., y Zúñiga-Jara, S., Aprendizaje basado en equipos con IF-AT: impacto y percepción en estudiantes universitarios, https://doi.org/10.4067/S0718-07052016000100016, Estudios Pedagógicos, 42(1), 255-269. (2018)

Salas, R., Enfoques de aprendizaje entre estudiantes universitarios, https://doi.org/doi:10.4067/S071807051998000100005, Estudios Pedagógicos, 24, 59-78 (1998)

Shaik, S.A., Almarzuqi, A., y otros cuatro autores, Assessing Saudi medical students learning approach using the revised two-factor study process questionnaire, https://doi.org/10.5116/ijme.5974.7a06, International Journal of Medical Education, 8, 292-296 (2017)

Vaughan, B., Confirmatory factor analysis of the Study Process Questionnaire in an Australian osteopathy student population, https://doi.org/https://doi.org/10.1016/j.ijosm.2016.03.001, International Journal of Osteopathic Medicine, 20, 62-67 (2016)

Vaughan, B., A rasch analysis of the Revised Study Process Questionnaire in an Australian osteopathy student cohort, https://doi.org/https://doi.org/10.1016/j.stueduc.2017.12.003, Studies in Educational Evaluation, 56, 144-153 (2018)

Vergara-Hernández, C., Simancas-Pallares, M. y Carbonell-Muñoz, Z., Psychometric properties of the revised two-factor Study Process Questionnaire R-SPQ-2f - Spanish version, https://doi.org/10.21676/2389783x.2744, Duazary, 16(2), 205218 (2019)

Wan, K., Cheung, G., y Chan, K., Prediction of students' use and acceptance of clickers by learning approaches: a crosssectional observational study, https://doi.org/10.3390/educsci7040091, Education Sciences, 7(4), 91 (2017)

Webster, B.J., Chan, W.S., y otros dos autores, Undergraduates' learning experience and learning process: quantitative evidence from the East. https://doi.org/10.1007/s10734-009-9200-6, Higher Education, 58, 375-386 (2009)

Wynn-Williams, K., Beatson, N., y Anderson, C., The impact of unstructured case studies on surface learners: a study of second-year accounting students, https://doi.org/10.1080/09639284.2016.1165125, Accounting Education, 25(3), 272-286 (2016)

Yáñez-Galleguillos, L.M., y Soria-Barreto, K., Reflexión de buenas prácticas docentes como eje de calidad en la educación universitaria: caso Escuela de Ciencias Empresariales de la Universidad Católica del Norte, https://doi.org/10.4067/s071850062017000500007, Formación Universitaria, 10(5), 59-68 (2017)

Yardimci, F., Bektaş, M., y otros cuatro autores, A study of the relationship between the study process, motivation resources, and motivation problems of nursing students in different educational systems, https://doi.org/https://doi.org/10.1016/j.nedt.2016.09.017, Nurse Education Today, 48, 13-18 (2017)

Zakariya, Y.F., Study approaches in higher education mathematics: investigating the statistical behavior of an instrument translated into Norwegian, https://doi.org/10.3390/educsci9030191, Education Sciences, 9(3), 1-10 (2019) 
